\title{
Spin precession and inverted Hanle effect in a semiconductor near a finite-roughness ferromagnetic interface
}

\author{
S. P. Dash, ${ }^{1}$ S. Sharma, ${ }^{2,3}$ J. C. Le Breton, ${ }^{2,4}$ J. Peiro, ${ }^{4}$ H. Jaffrès, ${ }^{4}$ J.-M. George, ${ }^{4}$ A. Lemaître, ${ }^{5}$ and R. Jansen ${ }^{3}$ \\ ${ }^{1}$ Department of Microtechnology and Nanoscience, Chalmers University of Technology, SE-41296, Göteborg, Sweden \\ ${ }^{2}$ Netherlands Foundation for Fundamental Research on Matter (FOM), NL-3502 GA Utrecht, The Netherlands \\ ${ }^{3}$ National Institute of Advanced Industrial Science and Technology (AIST), Spintronics Research Center, Tsukuba, Ibaraki 305-8568, Japan \\ ${ }^{4}$ Unité Mixte de Physique CNRS-Thales, F-91767 Palaiseau and Université Paris-Sud, F-91405 Orsay, France \\ ${ }^{5}$ Laboratoire de Photonique et Nanostructures, CNRS, F-91460 Marcoussis, France \\ (Received 7 April 2011; revised manuscript received 2 June 2011; published 4 August 2011)
}

\begin{abstract}
Although the creation of spin polarization in various nonmagnetic media via electrical spin injection from a ferromagnetic tunnel contact has been demonstrated, much of the basic behavior is heavily debated. It is reported here that, for semiconductor/ $\mathrm{Al}_{2} \mathrm{O}_{3} /$ ferromagnet tunnel structures based on $\mathrm{Si}$ or GaAs, local magnetostatic fields arising from interface roughness dramatically alter and even dominate the accumulation and dynamics of spins in the semiconductor. Spin precession in inhomogeneous magnetic fields is shown to reduce the spin accumulation up to tenfold, and causes it to be inhomogeneous and noncollinear with the injector magnetization. The inverted Hanle effect serves as the experimental signature. This interaction needs to be taken into account in the analysis of experimental data, particularly in extracting the spin lifetime $\tau_{s}$ and its variation with different parameters (temperature, doping concentration). It produces a broadening of the standard Hanle curve and thereby an apparent reduction of $\tau_{s}$. For heavily doped $n$-type $\mathrm{Si}$ at room temperature it is shown that $\tau_{s}$ is larger than previously determined, and a new lower bound of $0.29 \mathrm{~ns}$ is obtained. The results are expected to be general and to occur for spins near a magnetic interface not only in semiconductors but also in metals and organic and carbon-based materials including graphene, and in various spintronic device structures.
\end{abstract}

DOI: 10.1103/PhysRevB.84.054410

PACS number(s): 72.25.Hg, 72.25.Dc, 73.40.Gk, 85.75.-d

\section{INTRODUCTION}

The controlled creation of a nonequilibrium spin polarization in nonmagnetic materials is a central aspect of spintronics and plays a role in virtually all spin-based electronic nanostructures. ${ }^{1-3}$ In the spin valve, the best-known example of a metallic spintronic device consisting of two ferromagnetic layers separated by a thin nonmagnetic metal, spin information can be transmitted between the two ferromagnets via the spin accumulation in the spacer. This gives rise to giant magnetoresistance and exchange coupling, and allows one ferromagnet to exert a torque on the other. ${ }^{4-7}$ In a spin transistor, an example of a spin-based semiconductor device, spin information between ferromagnetic source and drain is transmitted via a semiconductor channel, ${ }^{8,9}$ making it possible to manipulate the spins during transit by a gate electric field. Understanding the physics of spins in nonmagnetic materials is thus crucial as it controls the overall behavior and performance of spin-based nanostructures. Although spin polarization has been electrically created in a variety of nonmagnetic materials, mostly via spin-polarized tunneling from a ferromagnetic contact, ${ }^{10-22}$ much of the basic physics is not understood. The magnitude and sign of the induced polarization are heavily debated, ${ }^{18,23-27}$ the variation with bias voltage and temperature is often puzzling, ${ }^{12,18,21,28,29}$ and unexpectedly short spin lifetimes are observed, for instance in the range of a few hundred picoseconds in graphene and doped $\mathrm{Si}$ at room temperature..$^{17,21}$

\section{A. Spins in proximity to a ferromagnetic interface}

Because spintronic nanostructures combine different materials (ferromagnets with nonmagnetic metals, semiconductors, and organic and carbon-based materials), a key question is to what extent the proximity to interfaces influences the spin accumulation and the spin dynamics. Dipolar fields from magnetic domain walls in a demagnetized $\mathrm{Ni}$ film have been reported to reduce the spin-dephasing time of optically excited carriers in $\mathrm{GaAs},{ }^{30,31}$ but the associated increase of the Hanle line width $(\sim 1 \mathrm{Oe})$ is small. Spin precession is also known to be affected by nuclear hyperfine fields. ${ }^{32-34}$ These are not related to the ferromagnetic interface and are typically relevant only at low temperature. In contrast, we demonstrate here a much more general mechanism (present even at room temperature and for homogeneously magnetized ferromagnetic electrodes) that has a surprisingly dramatic effect on spin accumulation and spin dynamics of carriers in a nonmagnetic medium near a magnetic interface. Specifically, inhomogeneous magnetostatic fields arising from finite interface roughness are shown to alter precession of spins in a semiconductor near the magnetic interface, dominate spin dynamics up to surprisingly large external fields as large as $1 \mathrm{kOe}$, and reduce the spin accumulation up to tenfold. We focus here on spin polarization created in semiconductors by injection of spins from a ferromagnetic tunnel contact. However, the phenomena described here should occur irrespective of the type of nonmagnetic material or the method used to create the spin accumulation, although the extent of the effect depends on the details of the system.

The magnetostatic fields near a ferromagnetic interface with finite roughness are sketched in Fig. 1 for the case of a sinusoidal interface profile with period $\lambda$. The magnetization of the ferromagnet is taken to lie in plane and point strictly along the global interface everywhere. This is a valid approximation for the soft magnetic thin films without significant interface anisotropy that we use here, as their magnetization can easily 

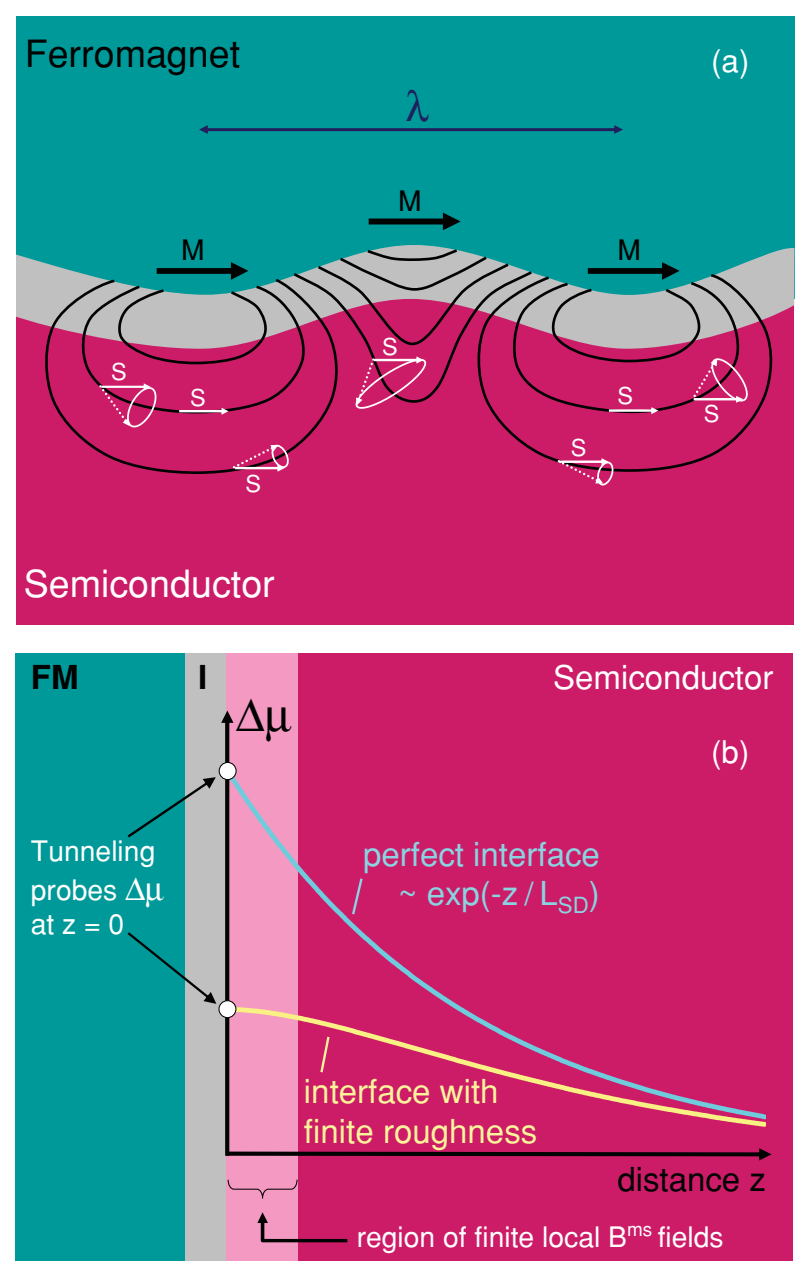

FIG. 1. (Color online) Illustration of local interface magnetic fields and their effect on spin precession in a semiconductor. (a) The inhomogeneous magnetostatic field near a ferromagnetic interface with finite roughness, sketched for a sinusoidal interface profile with period $\lambda$. Field lines are in black; the magnetization of the ferromagnet (black arrows) points strictly along the global interface plane everywhere. Spins are injected into the semiconductor with spin initially aligned with the magnetization of the ferromagnet (solid white arrows). In the local fields, the spins are precessing on different trajectories represented by dotted arrows and white ellipses. Also the strength of the local field and hence the precession frequency is spatially inhomogeneous. (b) Decay of the spin accumulation $\Delta \mu$ as a function of distance $z$ from the oxide/semiconductor interface for (i) a perfectly smooth interface (exponential decay with spin-diffusion length $L_{S D}$ ) and (ii) an interface with finite roughness. For the latter, the region in which the local magnetostatic fields $B^{m s}$ have an appreciable value is given in pink. Note that tunneling probes the value of $\Delta \mu$ at $z=0$

be saturated in a small in-plane magnetic field. While for an extended and perfectly flat, in-plane magnetized film the magnetostatic field would be zero outside the ferromagnet, in the presence of finite roughness there are local magnetostatic fields that penetrate into the nonmagnetic medium and influence the spins. Note that this is not only determined by the ferromagnet/tunnel barrier interface, but for thin films also by the roughness of the top surface of the ferromagnet, due to the long-range nature of magnetic fields. The magnetostatic fields are inhomogeneous in magnitude and direction, and change sign periodically. The magnitude of the fields scales with the roughness amplitude, and is linearly proportional to the magnetization $M_{s}$ of the ferromagnet. The strength of the field decays with distance $z$ from the interface on a length scale that, for periodic roughness, is $\operatorname{set}^{35}$ by the lateral roughness period $\lambda$. Under electrical spin injection from the ferromagnetic contact, a spin accumulation $\Delta \mu=\mu^{\uparrow}-\mu^{\downarrow}$ is induced, with $\mu^{\uparrow}\left(\mu^{\downarrow}\right)$ the electrochemical potential for electrons with majority (minority) spin. In the absence of roughness, $\Delta \mu$ decays exponentially as a function of distance $z$ from the injection interface [Fig. 1(a)], with a spin-diffusion length $L_{S D}$. However, for finite roughness spin precession is altered significantly in the region between $z=0$ and $z=\lambda$ where appreciable local magnetostatic fields exist, strongly reducing $\Delta \mu$. Even if $\lambda$ is shorter than $L_{S D}$, interfacial depolarization reduces $\Delta \mu$ over the full depth range [Fig. 1(b)] because spin diffusion connects all spins and dictates that spatial variations in spin density cannot exist on a length scale much smaller than $L_{S D}$. Hence, interfacial magnetostatic fields affect the spins to an effective depth of $L_{S D}$. Also note that by spin-polarized tunneling into the ferromagnet one probes the value of $\Delta \mu$ at $z=0$, where the reduction is strongest, as the spin accumulation right at the interface is most directly affected by the local magnetostatic fields.

\section{TUNNEL CONTACTS AND MEASUREMENTS}

We describe results for tunnel contacts on two different semiconductors ( $\mathrm{Si}$ and $\mathrm{GaAs}$ ). The device fabrication and electrical measurement techniques have been described previously. ${ }^{18,21}$ In brief, tunnel contacts of $\mathrm{Si} / \mathrm{Al}_{2} \mathrm{O}_{3} /$ ferromagnet have been prepared $^{21}$ by evaporation in ultrahigh vacuum using different ferromagnets (FMs) on $n$-type as well as $p$-type $\mathrm{Si}$ substrates (carrier density and resistivity of $1.8 \times 10^{19} \mathrm{~cm}^{-3}$ and $3 \mathrm{~m} \Omega \mathrm{cm}$ at room temperature for $n$-type $\mathrm{Si}$ with As doping, and $4.8 \times 10^{18} \mathrm{~cm}^{-3}$ and $11 \mathrm{~m} \Omega \mathrm{cm}$ at room temperature for $p$-type Si with B doping). The $\mathrm{GaAs} / \mathrm{Al}_{2} \mathrm{O}_{3} / \mathrm{Co}$ structures ${ }^{18}$ are grown by sputtering on $n$-type GaAs epilayers with a doping concentration of $5 \times 10^{18} \mathrm{~cm}^{-3}$ with a $15 \mathrm{~nm}$ heavily doped surface region $\left(2 \times 10^{19} \mathrm{~cm}^{-3}\right)$. All measurements are performed on contacts having dimensions of $100 \times 200 \mu \mathrm{m}^{2}(\mathrm{Si})$ and $15 \times 196 \mu \mathrm{m}^{2}$ (GaAs) in the so-called three-terminal geometry, ${ }^{18,21}$ probing the spin accumulation near a single ferromagnetic tunnel interface, thus using the same contact for spin injection and detection. Roughness characterization is presented in Appendix D.

\section{RESULTS}

\section{A. Spin precession in silicon near a ferromagnetic interface}

When spin-polarized electrons tunnel from the ferromagnet into the semiconductor, the injected spins initially point along the magnetization direction of the ferromagnet, taken to be along $x$. Ideally, in the absence of an external applied magnetic field $B^{\text {ext }}$ there is no Larmor spin precession, and a static, nonequilibrium spin accumulation is induced. The local magnetostatic fields $B^{m s}(x, y, z)$ modify this simple picture. 

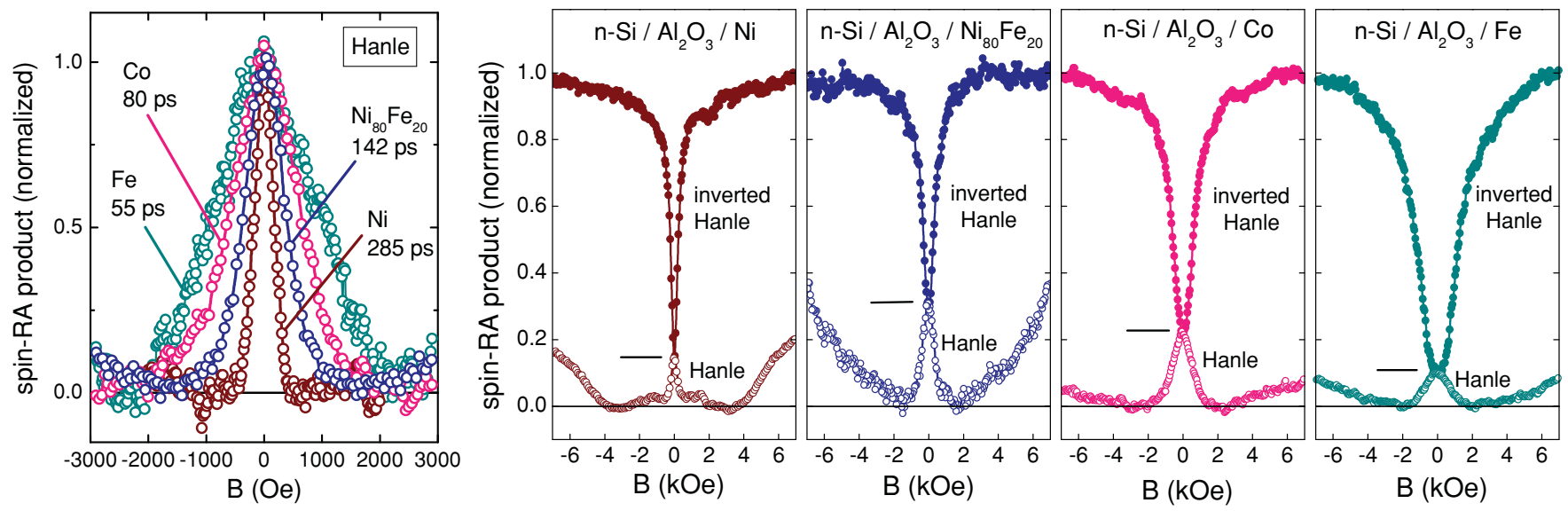

FIG. 2. (Color online) Spin accumulation and precession in $n$-type silicon near a ferromagnetic interface. Room temperature data for $n-\mathrm{Si} / \mathrm{Al}_{2} \mathrm{O}_{3} /$ ferromagnet junctions with $\mathrm{Ni}, \mathrm{Ni}_{80} \mathrm{Fe}_{20}, \mathrm{Co}$, or Fe electrode. The vertical axis gives the product of spin resistance and area (the "spin-RA product"), defined as $(\Delta V / I) \times$ area. The magnetic field is applied perpendicular to the interface plane (open symbols, Hanle), or parallel to the interface (solid symbols, inverted Hanle), with $V_{\mathrm{Si}}-V_{\mathrm{FM}}=+172 \mathrm{mV}$ (electron injection). In the left panel, Hanle curves for different FMs are normalized for better comparison of the linewidth, denoted by an effective time $1 / \omega$ representing the half width at half maximum of a fit to a Lorentzian (using $g=2$ ).

The spins are precessing in the total magnetic field that is composed of $B^{\text {ext }}$ and $B^{m s}(x, y, z)$. Since the latter is spatially inhomogeneous in direction and amplitude, the axis of spin precession and the precession frequency become spatially inhomogeneous. A full account of the consequences is given in Sec. IV, after description of the experimental data.

The spin accumulation is probed by establishing a constant tunnel current $I$ across the semiconductor $/ \mathrm{Al}_{2} \mathrm{O}_{3} / \mathrm{FM}$ tunnel contact, and measuring the change in voltage $\Delta V$ across that same tunnel contact as a function of $B^{\text {ext }}$. Since ${ }^{9,24,27,36}$ the tunnel resistance is directly proportional to $\Delta \mu$ (i.e., $\Delta V=P \Delta \mu / 2$ with $P$ the tunnel spin polarization associated with the $\mathrm{Al}_{2} \mathrm{O}_{3} / \mathrm{FM}$ interface) and $\Delta \mu$ is reduced by spin precession, the value of $\Delta V$ and its variation with $B^{\text {ext }}$ provide information about the spin dynamics. We start with $n$-type Si and conventional Hanle measurements (Fig. 2, left panel), with $B^{\text {ext }}$ applied along the $z$ axis (perpendicular to the interface and to the injected spins). A typical Hanle curve is observed, with a maximum voltage (and hence $\Delta \mu)$ at $B^{\text {ext }}=0$, and a gradual reduction with increasing external field due to spin precession. This is similar to Hanle data obtained previously, ${ }^{21}$ establishing that a nonequilibrium spin accumulation in the $\mathrm{Si}$ is induced by the injection of the spin-polarized tunnel current. Control experiments have previously excluded artifacts not related to spin injection. ${ }^{21}$ Previous work has also unambiguously established that the room-temperature spin polarization exists in the bulk bands of the Si rather than being enhanced by localized interface states (see the specific experiments reported in Fig. 3 of Ref. 21, and the observation of circularly polarized electroluminescence originating from $300 \mathrm{~nm}$ away from the injection interface in Si-based spin light-emitting diodes ${ }^{37}$ ). Despite this, we observe, as in previous work, ${ }^{21}$ spin signals for different ferromagnets (see below) in the range of $1-10 \mathrm{k} \Omega \mu \mathrm{m}^{2}$ and
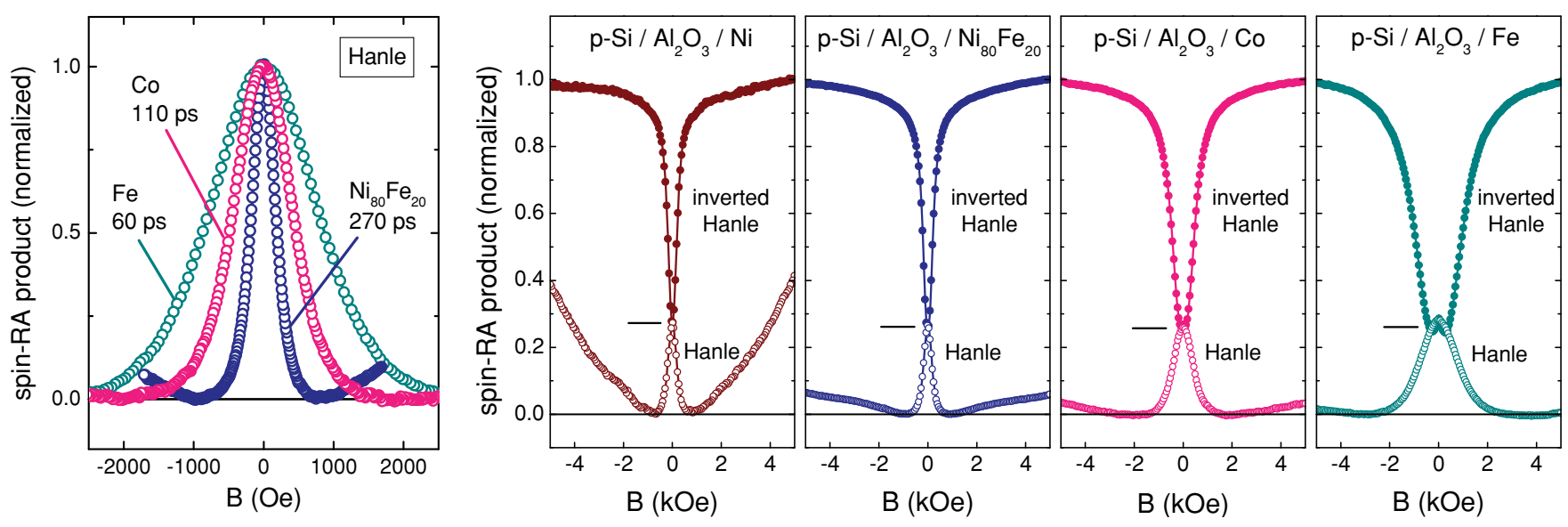

FIG. 3. (Color online) Spin accumulation and precession in $p$-type silicon near a ferromagnetic interface. Room temperature data for $p$-Si/ $\mathrm{Al}_{2} \mathrm{O}_{3}$ /ferromagnet junctions with $\mathrm{Ni}, \mathrm{Ni}_{80} \mathrm{Fe}_{20}$, $\mathrm{Co}$, or $\mathrm{Fe}$ electrode. The magnetic field is applied perpendicular to the interface plane (open symbols, Hanle), or parallel to the interface (solid symbols, inverted Hanle), with $V_{\mathrm{Si}}-V_{\mathrm{FM}}=-172 \mathrm{mV}$ (hole injection). In the left panel, Hanle curves for different FMs are normalized for better comparison of the linewidth, denoted by an effective time $1 / \omega$ representing the half width at half maximum of a fit to a Lorentzian (using $g=2$ ). 
thus larger than expected from theory, as noted before. ${ }^{21}$ The origin of this disagreement is still under discussion, but since enhancement by localized states has already been ruled out, ${ }^{21}$ there must be other enhancement factors that are not yet incorporated in existing theory. This is beyond the scope of the present work, which is concerned with the generic phenomena that affect the spin precession near a ferromagnetic interface and thereby the shape of the Hanle curve. Therefore we will not discuss here the factors that determine the overall magnitude of $\Delta \mu$, and show only normalized data. We did not find any correlation between the overall signal magnitude and the shape of the curves.

Let us now focus on the features that are due to the proximity of the interface with the ferromagnet. We find that the width of the Hanle curve depends on the ferromagnet used, i.e., the width increases from $\mathrm{Ni}$, to $\mathrm{Ni}_{80} \mathrm{Fe}_{20}$, to $\mathrm{Co}$, to $\mathrm{Fe}$, with a half width at half maximum (HWHM) of 200, 400, 710, and $1030 \mathrm{Oe}$, respectively. Conventionally, the Hanle curves are described $^{3,21}$ by a Lorentzian given by $\Delta \mu(B)=\Delta \mu(0) /[1+$ $\left.\left(\omega_{L} \tau_{s}\right)^{2}\right]$, where $\tau_{s}$ is the spin lifetime and $\omega_{L}$ is the Larmor frequency $\left(\omega_{L}=g \mu_{B} B / \hbar\right.$, where $g$ is the Landé $g$ factor, $\mu_{B}$ the Bohr magneton, and $\hbar$ Planck's constant divided by $2 \pi$ ). The width of the Hanle curve is then set solely by parameters of the semiconductor ( $\tau_{s}$ and $g$ ), which is inconsistent with our data. We attribute the experimental trend to modification of the spin dynamics near the FM interface due to local magnetostatic fields that arise for finite roughness. As shown in Sec. IV, this produces an artificial broadening of the Hanle curve that depends on the direction and magnitude of $B^{m s}$, which in turn is proportional to the magnetization $\left(M_{s}\right)$ of the FM. Indeed $\mu_{0} M_{s}$ at room temperature increases from $0.6 \mathrm{~T}$ for $\mathrm{Ni}$, to $0.9 \mathrm{~T}$ for $\mathrm{Ni}_{80} \mathrm{Fe}_{20}$, to $1.8 \mathrm{~T}$ for $\mathrm{Co}$, and to $2.2 \mathrm{~T}$ for $\mathrm{Fe}$.

\section{B. Inverted Hanle effect}

The above interpretation is proved by the following phenomenon, hereafter referred to as the inverted Hanle effect. It denotes the increase of the spin polarization in an applied (longitudinal) magnetic field [in analogy with the term Hanle effect, which gives a reduction of the spin polarization in an applied (transverse) magnetic field]. If $B^{\text {ext }}=0$, the spin accumulation will be reduced by precession in the $y$ and $z$ components of the local magnetostatic fields, which are orthogonal to the injected spins for a ferromagnet with magnetization along $x$. If now a nonzero $B_{x}^{\text {ext }}$ along $x$ is added and increased, the total magnetic field (vector sum of $B^{m s}$ and $B_{x}^{\text {ext }}$ ) rotates into the direction of the magnetization, thus reducing the angle between the injected spins and the axis of precession. The precession is suppressed, and an increase in the spin accumulation is expected as a function of $B_{x}^{\text {ext }}$. Indeed, the data in Fig. 2 show exactly this inverted Hanle effect for all FM electrodes. The smallest voltage (and hence $\Delta \mu$ ) is obtained for $B_{x}^{\text {ext }}=0$, while at large $B_{x}^{\text {ext }}$ the voltage across the contact saturates as spin precession in the local magnetostatic fields is fully eliminated. The saturation occurs at a larger field value for the ferromagnet with larger $M_{s}$, consistent with the outlined scenario. No dependence on the direction of the field in the $x-y$ plane was observed, as expected for polycrystalline magnetic films, for which roughness-induced magnetostatic fields should be isotropic. We conclude that application of an external in-plane magnetic field leads to a recovery of the spin accumulation, reaching the ideal value (that would be obtained without any precession) for large enough $B_{x}^{\text {ext }}$. The "true" value of the spin accumulation is thus given by the difference between the saturation signal of the inverted Hanle curve (large $\left.B_{x}^{\text {ext }}\right)$ and the minimum of the signal of the conventional Hanle curve with $B^{\text {ext }}$ along $z$. This difference has been normalized to 1 for all data presented. Importantly, the precession in local magnetostatic fields causes a significant reduction of the spin accumulation, with $\Delta \mu$ at $B^{\text {ext }}=0$ varying from $10 \%$ to $31 \%$ of the ideal value.

Note that an inhomogeneous spin accumulation can in principle also arise if the interface magnetization does not point along the global interface plane everywhere, as this would lead to inhomogeneity in the orientation of the spins that are injected. However, the in-plane magnetic coercivity of the magnetic films used here is 5-30 Oe and the films do not have any significant interface anisotropy. Therefore, the ferromagnet is homogeneously and fully magnetized along the external in-plane field well below 100 Oe. Hence, the spin injection is homogeneous and does not change for fields between 100 Oe and several kOe where the signal variation due to the inverted Hanle effect is observed. Even if some slight deviation of the interface magnetization from strictly in plane were present, this cannot account for the strongly reduced spin accumulation that is observed. This would require injection of carriers with spin pointing almost along the interface normal. This is not plausible and is inconsistent with the magnetic behavior of magnetic tunnel junctions prepared from the same materials. $^{38}$

Qualitatively similar results are obtained for tunnel contacts on $p$-type $\mathrm{Si}$ (Fig. 3). For all ferromagnets, a Hanle signal is observed at room temperature, consistent with our previous work on the creation of spin polarization in $p$-type $\mathrm{Si}^{21}$ For increasing $M_{s}$ the width of the Hanle curve increases, with HWHM of $200 \mathrm{Oe}(\mathrm{Ni}), 210 \mathrm{Oe}\left(\mathrm{Ni}_{80} \mathrm{Fe}_{20}\right), 515 \mathrm{Oe}(\mathrm{Co})$, and $950 \mathrm{Oe}(\mathrm{Fe})$, although the difference between $\mathrm{Ni}_{80} \mathrm{Fe}_{20}$ and pure Ni is small. For all devices an inverted Hanle curve is observed too, with a width and saturation field that increase systematically for FM electrodes with larger $M_{s}$. The induced $\Delta \mu$ at $B^{\text {ext }}=0$ is about $27 \%$ of the ideal value, but with less variation compared to the data for $n$-type $\mathrm{Si}$.

In principle one can still fit the Hanle curves with a Lorentzian and extract a time constant (given as labels in the left panels of Figs. 2 and 3). However, it should be treated as an effective time or a lower limit to the spin lifetime in the semiconductor, because interface magnetostatic fields are present and cause artificial broadening of the Hanle curve. Experimentally this situation is easily recognized if an inverted Hanle effect is observed. Nevertheless, the lower bound for the spin lifetime in the $n$-type Si we obtain ( $285 \mathrm{ps,} \mathrm{Ni} \mathrm{electrode)} \mathrm{is}$ already an improvement by a factor of 2 compared to previous work with $\mathrm{Ni}_{80} \mathrm{Fe}_{20}$ electrodes, ${ }^{21}$ and the true spin lifetime is expected to be larger.

\section{Spin precession in GaAs near a ferromagnetic interface}

A similar set of experiments was carried out on GaAs $/ \mathrm{Al}_{2} \mathrm{O}_{3} / \mathrm{Co}$ tunnel junctions at $T=10 \mathrm{~K}$ (Fig. 4). A Hanle signal is observed for $B^{\text {ext }}$ along $z$, establishing that 

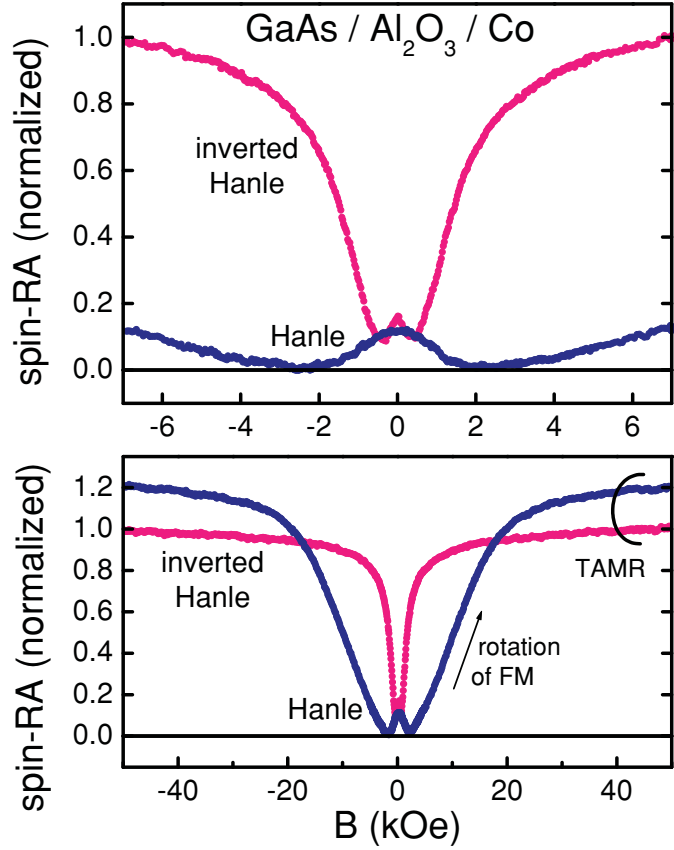

FIG. 4. (Color online) Spin accumulation and precession in $\mathrm{GaAs}$ near a ferromagnetic interface. Experimental data for $n$-type GaAs $/ \mathrm{Al}_{2} \mathrm{O}_{3} / \mathrm{Co}$ structures at $10 \mathrm{~K}$, for magnetic field applied perpendicular to the interface plane (blue, Hanle), or parallel to the interface (pink, inverted Hanle). Data at $V_{\mathrm{GaAs}}-V_{\mathrm{Co}}=+422 \mathrm{mV}$ (top panel) and $+580 \mathrm{mV}$ (bottom panel).

a nonequilibrium spin accumulation is created, although it has previously been proposed ${ }^{18}$ that the spins in these structures may accumulate primarily in localized states at or near the interface. Of course, spins in localized states also experience the magnetostatic fields from the nearby FM, consistent with the observation of the inverted Hanle effect (pink curves). The HWHM of the Hanle curve is 1070 Oe, slightly larger than in Si contacts with Co electrodes. The difference may be due to a different amplitude of the roughness, and/or the larger magnetization at low temperature. The effective time constant extracted from a fit to a Lorentzian is $1 / \omega=$ $55 \mathrm{ps}$, assuming a $g$ factor of 2 for electrons in localized interface states. The induced $\Delta \mu$ at $B^{\text {ext }}=0$ is $12 \%$ of the maximum spin accumulation. It should be noted that for spin accumulation in localized states in $\mathrm{GaAs} / \mathrm{Al}_{2} \mathrm{O}_{3} / \mathrm{Co}$ structures at low temperature, we cannot completely rule out that the behavior is caused by local magnetic (hyperfine) fields from nuclear spins, ${ }^{32}$ as previously studied with optical techniques in Voigt and Faraday geometry. ${ }^{33}$ However, given the results of the Si devices, it is highly likely that local magnetostatic fields arising from roughness are at the very least partly responsible for the behavior of the GaAs devices.

Additional insight is obtained from data at larger magnetic field (Fig. 4, bottom panel). When $B_{z}^{\text {ext }}$ is increased, the spin signal is first reduced due to the Hanle effect, but then sharply increases when the magnetization of the FM rotates out of plane, followed by a saturation of the spin accumulation at large fields when the magnetization, and hence the spins in the GaAs, is fully aligned with $B_{z}^{\text {ext }}$. Precession is then absent and the maximum $\Delta \mu$ is obtained. The value of $\Delta \mu$ thus achieved should be identical to the saturation value of the inverted Hanle curve, for which magnetization and spins in the GaAs and $B^{\text {ext }}$ all point along the $x$ axis and precession is absent too. A difference is, however, observed, attributed to anisotropy of the tunneling process. ${ }^{39-41}$ Apart from some quantitative differences, the results for GaAs- and Si-based devices are remarkably similar.

\section{MODEL}

First, we briefly address an important difference from the so-called orange-peel coupling that exists between two ferromagnets in layered structures with finite roughness. ${ }^{42,43}$ Due to the exchange interaction in a ferromagnet, it experiences only an average magnetostatic field from the other ferromagnet, reducing the effective coupling field to a few tens of oersteds. In contrast, in a nonmagnetic semiconductor the spins in different locations near the ferromagnetic electrode can precess independently, and sense the full local strength of the magnetostatic field, rather than an average. Hence, the relevant magnetic field scale for spins accumulating in a nonmagnetic material near a ferromagnet with finite roughness is much larger than that of orange-peel coupling.

The model that captures the basic physics of spin accumulation and precession near a ferromagnetic interface and correctly describes the salient experimental behavior starts from the equation ${ }^{3,44}$ for spin dynamics of an ensemble of spins in a nonmagnetic host:

$$
\frac{\partial \mathbf{S}}{\partial t}=\mathbf{S} \times \omega_{L}+D \nabla^{2} \mathbf{S}-\frac{\mathbf{S}}{\tau_{s}},
$$

where $\mathbf{S}$ is the spin density and $\omega_{L}=\left(\omega_{x}, \omega_{y}, \omega_{z}\right)=$ $\left(g \mu_{B} / \hbar\right)\left(B_{x}, B_{y}, B_{z}\right)$. Terms on the right-hand side describe, respectively, spin precession, spin diffusion ( $D$ is the diffusion constant), and spin relaxation. Spin drift has been neglected. We seek a solution for a homogeneous $B^{\text {ext }}$ plus inhomogeneous magnetostatic fields near the FM interface: $B_{i}=B_{i}^{\mathrm{ext}}+B_{i}^{m s}(x, y, z)$, with $i=x, y, z$.

In the limit where the spin-diffusion length $L_{S D}$ is small compared to the roughness period $\lambda$, the spin-diffusion term in Eq. (1) can be neglected. This provides an analytical solution that is strictly correct when electrons are sufficiently localized for gradients in the spin density to be sustained on the length scale of $\lambda$. This applies to the case of spin accumulation in localized states (as in the GaAs devices ${ }^{18}$ ). It is not strictly valid for mobile electrons since spin diffusion tends to average out the inhomogeneity of the spin density (in our Si devices $L_{S D}$ is $^{21}$ at least a few $100 \mathrm{~nm}$, while $\lambda$ is estimated to be $20-60 \mathrm{~nm}$; see Appendix D). The net result is a more homogeneous spin density, but with a reduced value. Although a rigorous, but cumbersome, numerical treatment including spin diffusion can be done, we can expect that the value of the spin accumulation with spin diffusion is comparable to the spatial average of the inhomogeneous spin density that is calculated without spin diffusion. We therefore average the spin density over the $x-y$ plane, finding that the basic experimental trends of the $\mathrm{Si}$ and $\mathrm{GaAs}$ devices are reproduced. Without spin diffusion, the general steady state solution for the $x, y$, and $z$ components of 

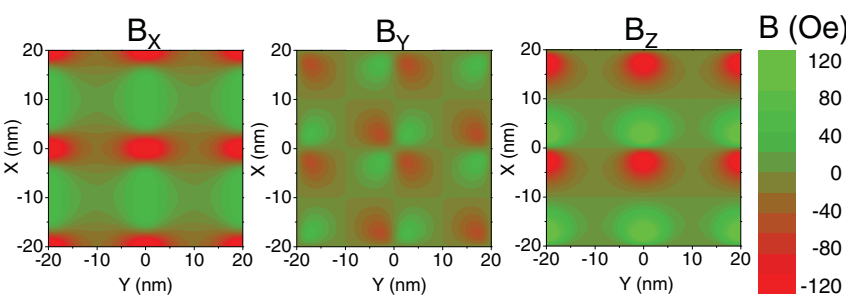

FIG. 5. (Color online) Profiles of magnetostatic fields near a ferromagnetic interface with finite roughness. Calculated $B_{x}, B_{y}$, and $B_{z}$ components of the field versus position in the $x-y$ plane at $5 \mathrm{~nm}$ distance from the interface, represented by a two-dimensional square array of magnetic dipoles pointing along the $x$ axis (period $\lambda=$ $20 \mathrm{~nm}$, central dipole at $x=y=0$ ). The dipole strength $\mu$ is such that $\mu_{0} \mu / 4 \pi=2 \mathrm{~T} \mathrm{~nm}^{3}$.

the spin density is ${ }^{44}$ (see also Appendix A)

$$
\begin{gathered}
S_{x}=S_{0}\left\{\frac{\omega_{x}^{2}}{\omega_{L}^{2}}+\left(\frac{\omega_{y}^{2}+\omega_{z}^{2}}{\omega_{L}^{2}}\right)\left(\frac{1}{1+\left(\omega_{L} \tau_{s}\right)^{2}}\right)\right\}, \\
S_{y}=S_{0}\left\{\frac{\omega_{x} \omega_{y}}{\omega_{L}^{2}}-\left(\frac{\omega_{x} \omega_{y}}{\omega_{L}^{2}}+\omega_{z} \tau_{s}\right)\left(\frac{1}{1+\left(\omega_{L} \tau_{s}\right)^{2}}\right)\right\}, \\
S_{z}=S_{0}\left\{\frac{\omega_{x} \omega_{z}}{\omega_{L}^{2}}-\left(\frac{\omega_{x} \omega_{z}}{\omega_{L}^{2}}-\omega_{y} \tau_{s}\right)\left(\frac{1}{1+\left(\omega_{L} \tau_{s}\right)^{2}}\right)\right\},
\end{gathered}
$$

where $\omega_{L}^{2}=\omega_{x}^{2}+\omega_{y}^{2}+\omega_{z}^{2}$ and $\omega_{i}=\omega_{i}^{\mathrm{ext}}+\omega_{i}^{m s}(x, y, z)$. Importantly, as $\omega^{m s}$ is spatially inhomogeneous, the spin density is too. Second, while the injected tunnel electrons have spin along the $x$ axis, for nonzero $B^{m s}$, the steady state spin density has $x, y$, and $z$ components and is thus generally noncollinear with the magnetization of the ferromagnetic injector (pointing strictly along $x$ ). Third, without external field there is no suppression of the spin polarization if $\omega_{y}^{m s}=\omega_{z}^{m s}=$ $0\left(S_{x}=S_{0}\right.$ and $\left.S_{y}=S_{z}=0\right)$, whereas $S_{x}<S_{0}$ in the presence of magnetostatic fields with components orthogonal to the injected spins (i.e., when $\omega_{y}^{m s} \neq 0$ and/or $\omega_{z}^{m s} \neq 0$ ). Hereafter we shall focus on the $S_{x}$ component, since in electrical detection using the same ferromagnetic tunnel contact only this component is relevant (the tunnel resistance is proportional to the projection of the spin accumulation onto the detector magnetization).

To evaluate $B^{m s}$ of a FM with finite roughness, we describe it as a two-dimensional square array of magnetic dipoles pointing along $x$ and calculate the magnetostatic fields (see Fig. 5). This gives an inhomogeneous pattern with all three field components present. Alternatively, for one-dimensional roughness an exact expression ${ }^{45}$ for $B^{m s}$ in terms of roughness amplitude and $M_{s}$ is given in Appendix C. From this and the measured roughness of our structures (Appendix D), we find that the strength of the magnetostatic fields can easily be in the range of $1 \mathrm{kOe}$ to 100 Oe up to a distance of $10 \mathrm{~nm}$ from the interface.

Figure 6 shows spatial maps of $S_{x}$ obtained from Eq. (2) using the magnetostatic fields at a distance of $5 \mathrm{~nm}$ from a square array of dipoles, and $\tau_{s}=1 \mathrm{~ns}$. For $B^{\text {ext }}=0$, the left panel shows regions with strongly reduced spin density (blue) due to precession in the local ( $y$ and $z$ components of the) magnetostatic fields, and regions where precession is absent and the maximum spin accumulation is present (red). When an external magnetic field is added along $z$ (Hanle configuration, top row) the precession is enhanced everywhere and the spin density is gradually reduced. In contrast, when $B^{\text {ext }}$ is applied along $x$ (inverted Hanle configuration, bottom row), everywhere the spin density increases toward its maximum value (red) as precession in $B^{m s}$ is suppressed. By averaging these maps over the $x-y$ plane, we obtain the variation of the average spin density as a function of $B^{\text {ext }}$ (right two panels). This qualitatively reproduces the experimental data: (i) the spin density at $B^{\text {ext }}=0$ is reduced from its maximum, (ii) there is an inverted Hanle effect, (iii) the width of the conventional Hanle curve is broadened as compared to the situation without magnetostatic fields, which would produce a Lorentzian with $\tau_{s}=1 \mathrm{~ns}$ (shown in green, with amplitude adjusted for easy comparison), and (iv) for increasing amplitude of $B^{m s}$ (larger dipole moment, bottom panel), the width of the Hanle curve increases, and the inverted Hanle curve and the reduction of the spin density at $B^{\text {ext }}=0$ become more pronounced. We conclude that, despite the neglect of spin diffusion, the model agrees well with the experimental observations and captures the basic physics.

Above we have included $B^{m s}$ only in $\omega_{L}$ of the precession term of Eq. (1), without changing $\tau_{s}$ in the last term. That is, we have modeled the phenomenon as being due to changes in the axis and frequency of the (locally) coherent precession of the ensemble spin polarization, modifying the measured time average of the spin density, and leading to artificial broadening of the Hanle curve and thereby an apparent shortening of the spin lifetime. In addition, the spatial inhomogeneity of the magnetostatic fields leads to decoherence and further broadening. Let us now consider whether the inhomogeneous magnetic fields have an effect on $\tau_{s}$. For localized electrons there is no effect on $\tau_{s}$. However, mobile electrons near a FM interface moving through a spatially inhomogeneous magnetostatic field experience this as a field fluctuating in time. This is distinct from D'yakonov-Perel' spin relaxation, where the fluctuation is due to changes of the momentum, rather than the location in real space that is relevant here. The associated time scale is given by $\tau^{m s}=\lambda / 4 v$, where $v$ is the carrier velocity and $\lambda / 4$ the length scale over which the field changes significantly. Since electrons with different trajectories acquire a different spin-precession phase and transport is random, this causes irreversible dephasing of the ensemble spin. Considering an electron moving parallel to the interface and typical parameters $(\lambda<100 \mathrm{~nm}$ and $v=10^{5} \mathrm{~m} / \mathrm{s}$ for electrons in $\mathrm{Si}$ ), $\tau^{\mathrm{ms}}$ is below $1 \mathrm{ps}$ and thus smaller than the spin-precession period for practical fields $\left(1 / \omega_{L} \geqslant 5\right.$ ps for $\left.B \leqslant 1 \mathrm{~T}\right)$. Hence, we are in the regime of motional narrowing ${ }^{46}$ and the associated spin-dephasing time is given ${ }^{46}$ by $T_{2}^{m s}=1 / \Omega_{a v}^{2} \tau^{m s}$, where $B_{a v}=\hbar \Omega_{a v} / g \mu_{B}$ is the average amplitude of the magnetostatic field. We thus have $1 / T_{2}=1 / T_{2}^{\text {bulk }}+1 / T_{2}^{m s}$, where $T_{2}^{\text {bulk }}$ is the regular spin-dephasing time in the absence of local magnetostatic fields. For $B_{a v} \leqslant 100 \mathrm{mT}$ and $\tau^{m s}=1 \mathrm{ps}$ we obtain $T_{2}^{m s} \geqslant$ $3 \mathrm{~ns}$. This is larger than the spin lifetimes we observe, and we therefore described the spin dynamics with a single spin lifetime, including the magnetostatic fields only in the coherent precession term of Eq. (1). In other situations, especially when 


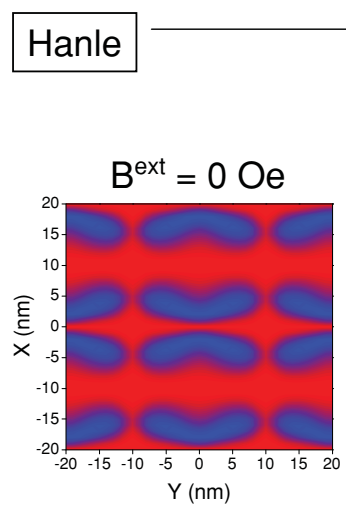

Inverted Hanle
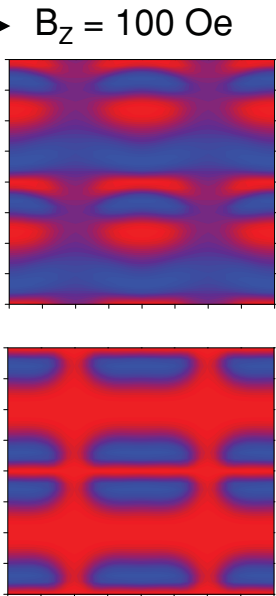

$B_{x}=100 \mathrm{Oe}$
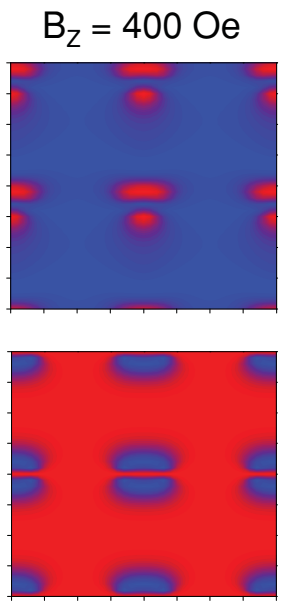

$\mathrm{B}_{\mathrm{X}}=400 \mathrm{Oe}$
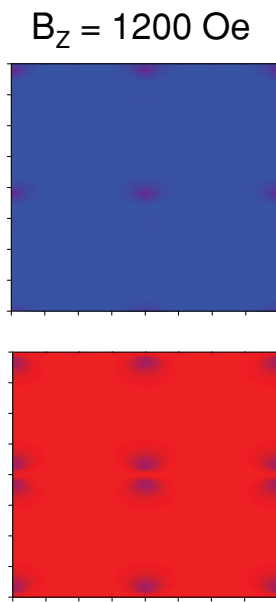

$\mathrm{B}_{\mathrm{x}}=1200 \mathrm{Oe}$

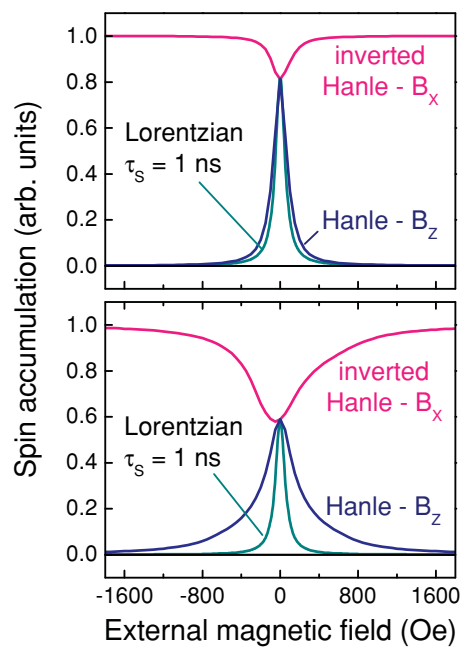

FIG. 6. (Color online) Calculated spin accumulation near an interface with local magnetostatic fields. Maps of the $S_{x}$ component of the spin density in the semiconductor versus position in the $x-y$ plane parallel to the interface, for different values of external magnetic field applied along the $z$ axis (Hanle configuration, top row), or along the $x$ axis parallel to the magnetization of the ferromagnetic injector (inverted Hanle configuration, bottom row). Red color corresponds to the maximum spin density (without any precession), blue to zero spin accumulation.

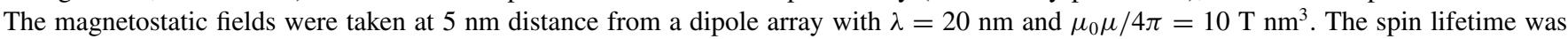
set to $1 \mathrm{~ns}$. Right panels show the resulting Hanle (blue) and inverted Hanle (pink) curves for external applied magnetic field along the $z$ or $x$ axis, respectively, calculated by averaging the inhomogeneous spin density over the $x-y$ plane. The dipole strength is such that $\mu_{0} \mu / 4 \pi=2$ (top panel) or $10 \mathrm{~T} \mathrm{~nm}^{3}$ (bottom panel). Also shown in green are pure Lorentzian line shapes for the same $1 \mathrm{~ns}$ spin lifetime, with the peak amplitude scaled for easy comparison.

$T_{2}^{\text {bulk }}$ is large, this source of dephasing may be of importance or even become limiting.

\section{IMPLICATIONS}

Perhaps the most immediate implication relates to the spin lifetime in $\mathrm{Si}$, which was previously ${ }^{21}$ extracted from Hanle data as about $140 \mathrm{ps}$ for heavily doped $n$-type $\mathrm{Si}$ at room temperature. We observe a clear inverted Hanle effect, the experimental signature that the conventional Hanle curve is artificially broadened by interfacial magnetostatic fields from the FM, such that a fit to a Lorentzian will underestimate the spin lifetime. Indeed, a new lower bound to the spin lifetime for $n$-type $\mathrm{Si}$ at room temperature was determined here (285 ps), and the actual spin lifetime must be still larger than that. The artificial broadening may also obscure the intrinsic variation of the spin lifetime with parameters such as temperature and doping concentration, and should thus be considered to allow a meaningful discussion of trends. Similar implications may be expected for other material systems, particularly when spins accumulate close to the FM, such as in a single layer of graphene. More generally, the phenomena described here will appear for spin accumulation near ferromagnetic interfaces created by any means (such as optical injection, electrical injection by tunneling, diffusive or ballistic transport, or via spin Hall and other spin-orbit effects), in different device geometries (two-, three-, and nonlocal four-terminal devices), and for various nonmagnetic materials (metals, semiconductors, and organic and carbon-based systems). The roughness-induced local magnetostatic fields and the resulting inhomogeneity of the spin accumulation and precession should be taken into account in the analysis of spin transport and dynamics, and may affect the properties and performance of spintronic devices.

\section{ACKNOWLEDGMENTS}

The authors are grateful to A. Fert for continuing support, discussions, and feedback, to M. Tran for his contribution to the GaAs device development, and to M. Yamamoto and $\mathrm{T}$. Yorozu for the roughness characterization by AFM. This work was financially supported by the Netherlands Foundation for Fundamental Research on Matter (FOM).

\section{APPENDIX A: STEADY STATE SPIN ACCUMULATION FOR ARBITRARY MAGNETOSTATIC FIELD}

A local magnetostatic field $B^{m s}$ arising from interface roughness adds to the external applied magnetic field $B^{\text {ext }}$ and thereby changes the local axis of coherent spin precession, as well as the precession frequency. To describe this, we start from the equation ${ }^{3}$ for spin dynamics of an ensemble of spins in a nonmagnetic host:

$$
\frac{\partial \mathbf{S}}{\partial t}=\mathbf{S} \times \omega_{L}+D \nabla^{2} \mathbf{S}-\frac{\mathbf{S}}{\tau_{s}},
$$

where $\mathbf{S}$ is the spin density and $\omega_{L}=\left(\omega_{x}, \omega_{y}, \omega_{z}\right)=$ $\left(g \mu_{B} / \hbar\right)\left(B_{x}, B_{y}, B_{z}\right)$. Terms on the right-hand side describe, respectively, spin precession, spin diffusion ( $D$ is the diffusion constant), and spin relaxation. We have neglected spin drift. 
The $x, y$, and $z$ components of the spin density are explicitly written as

$$
\begin{aligned}
\frac{\partial S_{x}}{\partial t} & =S_{y} \omega_{z}-S_{z} \omega_{y}+D \nabla^{2} S_{x}-\frac{S_{x}}{\tau_{s}}, \\
\frac{\partial S_{y}}{\partial t} & =S_{z} \omega_{x}-S_{x} \omega_{z}+D \nabla^{2} S_{y}-\frac{S_{y}}{\tau_{s}}, \\
\frac{\partial S_{z}}{\partial t} & =S_{x} \omega_{y}-S_{y} \omega_{x}+D \nabla^{2} S_{z}-\frac{S_{z}}{\tau_{s}} .
\end{aligned}
$$

If spin diffusion can be neglected (for spin-diffusion length $L_{S D}$ much smaller than the period $\lambda$ of the roughness), and the boundary conditions at $t=0$ are

$$
\begin{aligned}
& S_{x}(t=0)=A, \\
& S_{y}(t=0)=0, \\
& S_{z}(t=0)=0,
\end{aligned}
$$

then the analytic solutions for arbitrary magnetic field are given by

$$
\begin{aligned}
S_{x}(t)= & A\left\{\frac{\omega_{x}^{2}+\left(\omega_{y}^{2}+\omega_{z}^{2}\right) \cos \left(\omega_{L} t\right)}{\omega_{L}^{2}}\right\} \exp \left(-t / \tau_{s}\right), \\
S_{y}(t)= & A\left\{\frac{\omega_{x} \omega_{y}-\omega_{x} \omega_{y} \cos \left(\omega_{L} t\right)-\omega_{L} \omega_{z} \sin \left(\omega_{L} t\right)}{\omega_{L}^{2}}\right\} \\
& \times \exp \left(-t / \tau_{s}\right), \\
S_{z}(t)= & A\left\{\frac{\omega_{x} \omega_{z}-\omega_{x} \omega_{z} \cos \left(\omega_{L} t\right)+\omega_{L} \omega_{y} \sin \left(\omega_{L} t\right)}{\omega_{L}^{2}}\right\} \\
& \times \exp \left(-t / \tau_{s}\right),
\end{aligned}
$$

with $\omega_{L}^{2}=\omega_{x}^{2}+\omega_{y}^{2}+\omega_{z}^{2}$. These expressions describe the time evolution of a packet of spins initially polarized along the $x$ axis at $t=0$. The steady state spin polarization under continuous injection is proportional to the time integral $\int_{0}^{\infty} S_{i}(t) d t$, which yields

$$
\begin{array}{r}
S_{x}=S_{0}\left\{\frac{\omega_{x}^{2}}{\omega_{L}^{2}}+\left(\frac{\omega_{y}^{2}+\omega_{z}^{2}}{\omega_{L}^{2}}\right)\left(\frac{1}{1+\left(\omega_{L} \tau_{s}\right)^{2}}\right)\right\} \\
S_{y}=S_{0}\left\{\frac{\omega_{x} \omega_{y}}{\omega_{L}^{2}}-\left(\frac{\omega_{x} \omega_{y}}{\omega_{L}^{2}}+\omega_{z} \tau_{s}\right)\left(\frac{1}{1+\left(\omega_{L} \tau_{s}\right)^{2}}\right)\right\}, \\
S_{z}=S_{0}\left\{\frac{\omega_{x} \omega_{z}}{\omega_{L}^{2}}-\left(\frac{\omega_{x} \omega_{z}}{\omega_{L}^{2}}-\omega_{y} \tau_{s}\right)\left(\frac{1}{1+\left(\omega_{L} \tau_{s}\right)^{2}}\right)\right\},
\end{array}
$$

where $S_{0}$ is the spin polarization in the absence of any magnetic field, and $\omega_{i}=\omega_{i}^{\mathrm{ext}}+\omega_{i}^{m s}(x, y, z)$. Equation (A11) can be written in terms of the solid angle $\theta$ between injected spins and magnetic field vector, as was done previously ${ }^{44}$ for optical excitation:

$$
S_{x}=S_{0}\left\{\cos ^{2}(\theta)+\left(\frac{\sin ^{2}(\theta)}{1+\left(\omega_{L} \tau_{s}\right)^{2}}\right)\right\} .
$$

Without an external applied magnetic field, the spin polarization is determined exclusively by the local magnetostatic fields due to roughness:

$$
\begin{aligned}
S_{x}= & S_{0}\left\{\frac{\left(\omega_{x}^{m s}\right)^{2}}{\left(\omega_{L}^{m s}\right)^{2}}+\left(\frac{\left(\omega_{y}^{m s}\right)^{2}+\left(\omega_{z}^{m s}\right)^{2}}{\left(\omega_{L}^{m s}\right)^{2}}\right)\right. \\
& \left.\times\left(\frac{1}{1+\left(\omega_{L}^{m s} \tau_{s}\right)^{2}}\right)\right\},
\end{aligned}
$$

where $\left(\omega_{L}^{m s}\right)^{2}=\left(\omega_{x}^{m s}\right)^{2}+\left(\omega_{y}^{m s}\right)^{2}+\left(\omega_{z}^{m s}\right)^{2}$. Note that the reduction of the spin polarization depends, in general, on the strength as well as on the orientation of the local magnetostatic fields. However, in the limit $\omega_{L}^{m s} \tau_{s} \gg 1$, only the orientation of the field is relevant and $S_{x}$ becomes independent of the field strength (and hence independent of the magnetization of the ferromagnet).

\section{APPENDIX B: HANLE CURVES IN THE PRESENCE OF LOCAL MAGNETOSTATIC FIELDS}

The effect of the different components of the local magnetostatic field on the Hanle curves was calculated from Eq. (A11) using, for illustrative purposes, magnetostatic fields $B^{m s}$ pointing purely along either the $x, y$, or $z$ axis. The field strength is taken to have a periodic spatial variation $\omega_{i}^{m s}=\omega_{0} \cos (2 \pi x / \lambda)$, and the spin polarization was averaged in space over a full period $\lambda$. The resulting Hanle curves $\left(B^{\text {ext }}\right.$ along $z$ ) and inverted Hanle curves ( $B^{\text {ext }}$ along $x$ ) are shown in Fig. 7. We see the following.

(i) $B^{m s}$ along $x$, parallel to the injected spins. The Hanle curve is broadened, but there is no inverted Hanle signal and no reduction of the spin accumulation at zero external field.

(ii) $B^{m s}$ along $y$, orthogonal to the injected spins. The Hanle curve is broadened, and there is an inverted Hanle signal and a reduction of the spin accumulation at zero external field.

(iii) $B^{m s}$ along $z$, orthogonal to the injected spins. There is an inverted Hanle signal and a reduction of the spin accumulation at zero external field, while the Hanle curve is broadened, as well as split into two components, corresponding to locations with $B_{z}^{m s}$ aligned or antialigned with the external field $B_{z}^{\text {ext }}$.

\section{APPENDIX C: LOCAL MAGNETOSTATIC FIELDS NEAR A FERROMAGNET WITH ONE-DIMENSIONAL ROUGHNESS}

The pattern and magnitude of the local magnetostatic fields for a ferromagnet with one-dimensional roughness can be obtained via a Fourier transform. ${ }^{45}$ Taking the surface height to vary along the $x$ axis with period $\lambda$, a square height profile with peak-to-peak height $h$, and magnetization pointing along the $x$ direction, we have ${ }^{45}$

$$
\begin{aligned}
B_{x}^{m s}(x, z)= & \mu_{0} M_{s}\left(\frac{h}{2}\right) \sum_{n=1}^{\infty} q_{n} F\left(q_{n}\right) \\
& \times \exp \left(-q_{n} z\right) \sin \left(q_{n} x-\pi / 2\right), \\
& B_{y}^{m s}(x, z)=0,
\end{aligned}
$$




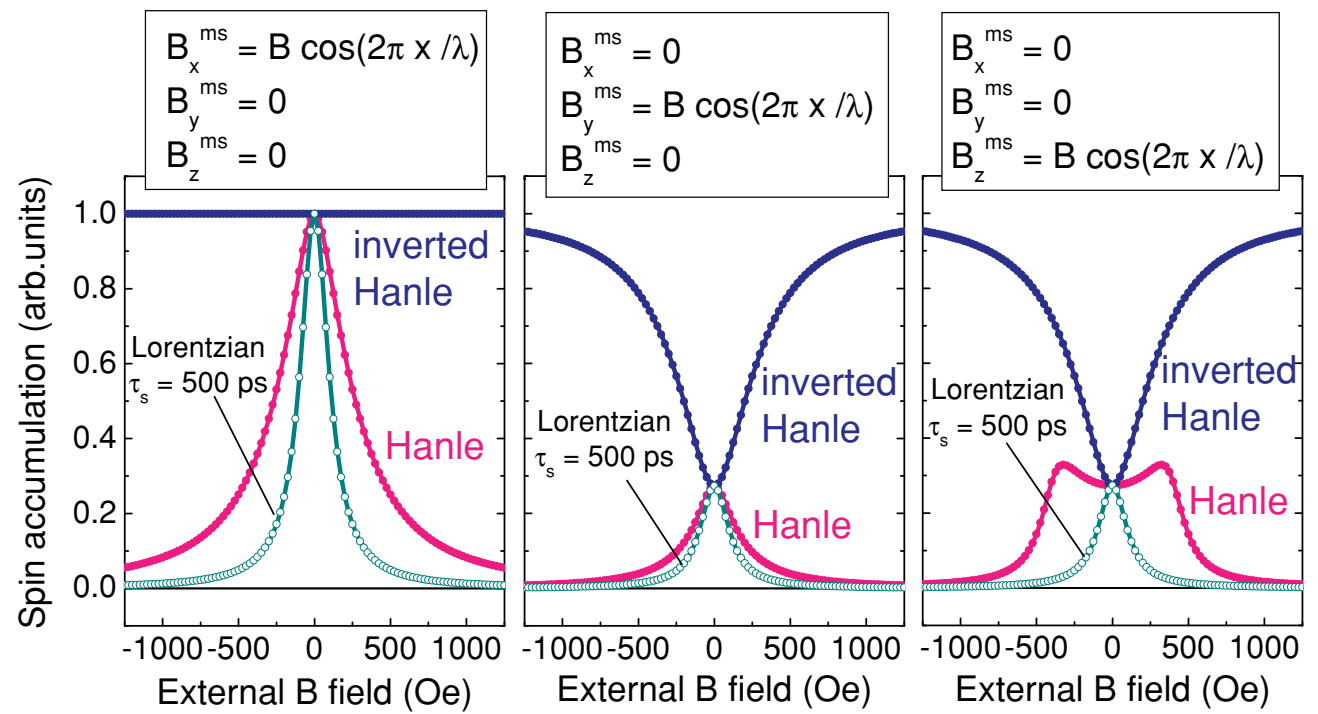

FIG. 7. (Color online) Hanle (pink) and inverted Hanle (blue) curves for external applied magnetic field $B^{\text {ext }}$ along $z$ or $x$ axis, respectively, calculated using Eq. (A11). Included are magnetostatic fields $B^{m s}$ pointing purely along either the $x, y$, or $z$ axis, as indicated, and with a strength that has a simple sinusoidal spatial variation. The spin lifetime $\tau_{s}$ was set to $500 \mathrm{ps}$. Also shown in green for the same $500 \mathrm{ps}$ spin lifetime are the pure Lorentzian curves, with the amplitude adjusted for easy comparison.

$$
\begin{aligned}
B_{z}^{m s}(x, z)= & \mu_{0} M_{s}\left(\frac{h}{2}\right) \sum_{n=1}^{\infty} q_{n} F\left(q_{n}\right) \\
& \times \exp \left(-q_{n} z\right) \cos \left(q_{n} x-\pi / 2\right),
\end{aligned}
$$

where $q_{n}=2 \pi n / \lambda$, and

$$
F\left(q_{n}\right)=\frac{\sin \left(q_{n} \lambda / 4\right)}{\left(q_{n} \lambda / 4\right)} \frac{\sinh \left(q_{n} h / 2\right)}{\left(q_{n} h / 2\right)} .
$$

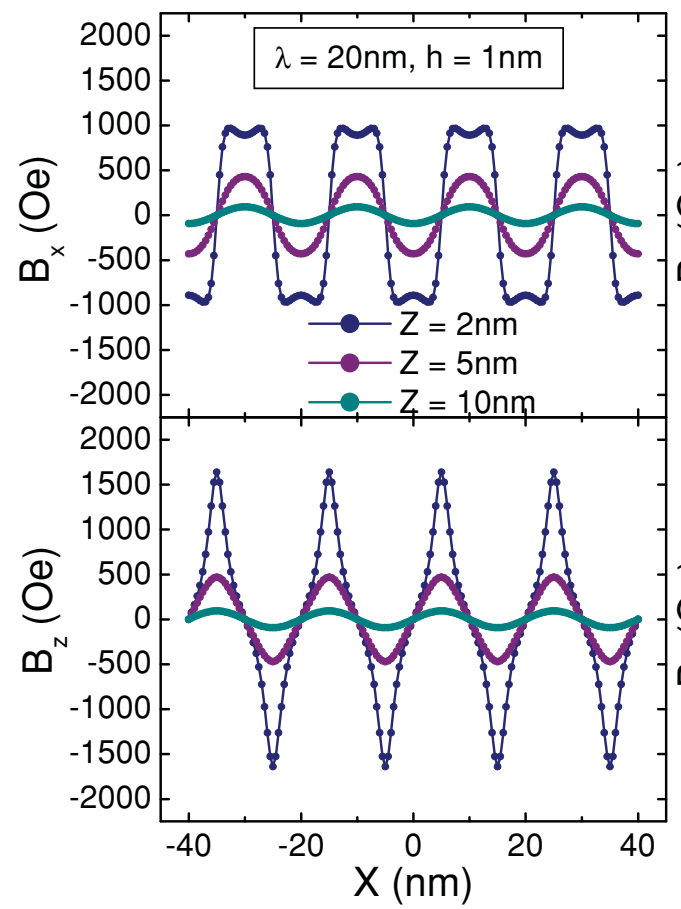

The fields for ferromagnetic Fe (having $\mu_{0} M_{s}=2.2 \mathrm{~T}$ ) are shown in Fig. 8. We find that the decay of the field strength with distance from the ferromagnet is determined by $\lambda$, and that for reasonable parameters the local magnetostatic fields can easily be in the range of $1 \mathrm{kOe}$ to $100 \mathrm{Oe}$ up to a distance of $10 \mathrm{~nm}$ away from the surface of the ferromagnet, thus having a significant impact on the spin accumulation and spin dynamics near the interface.

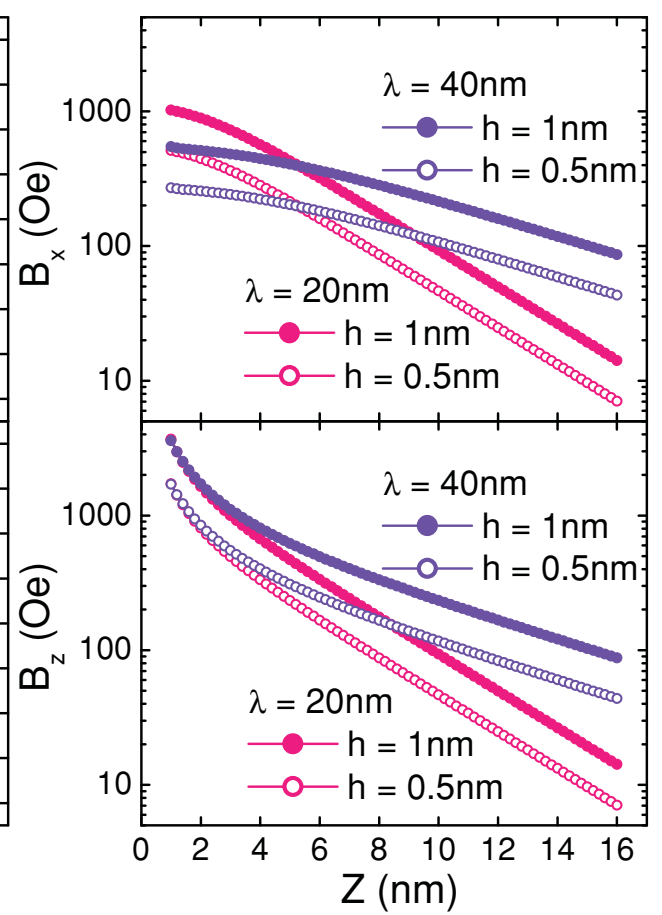

FIG. 8. (Color online) Left panels: Calculated strengths of the $B_{x}$ (top) and $B_{z}$ (bottom) components of the local magnetostatic field as a function of lateral $x$ position at different distances $z$ from a ferromagnetic Fe surface with one-dimensional roughness, for $\lambda=20 \mathrm{~nm}$ and roughness amplitude $h=1 \mathrm{~nm}$. Right panels: The same, but now as a function of distance $z$ at fixed $x$ position, for $\lambda=20$ or $40 \mathrm{~nm}$ and roughness amplitude $h=1$ or $0.5 \mathrm{~nm}$. 

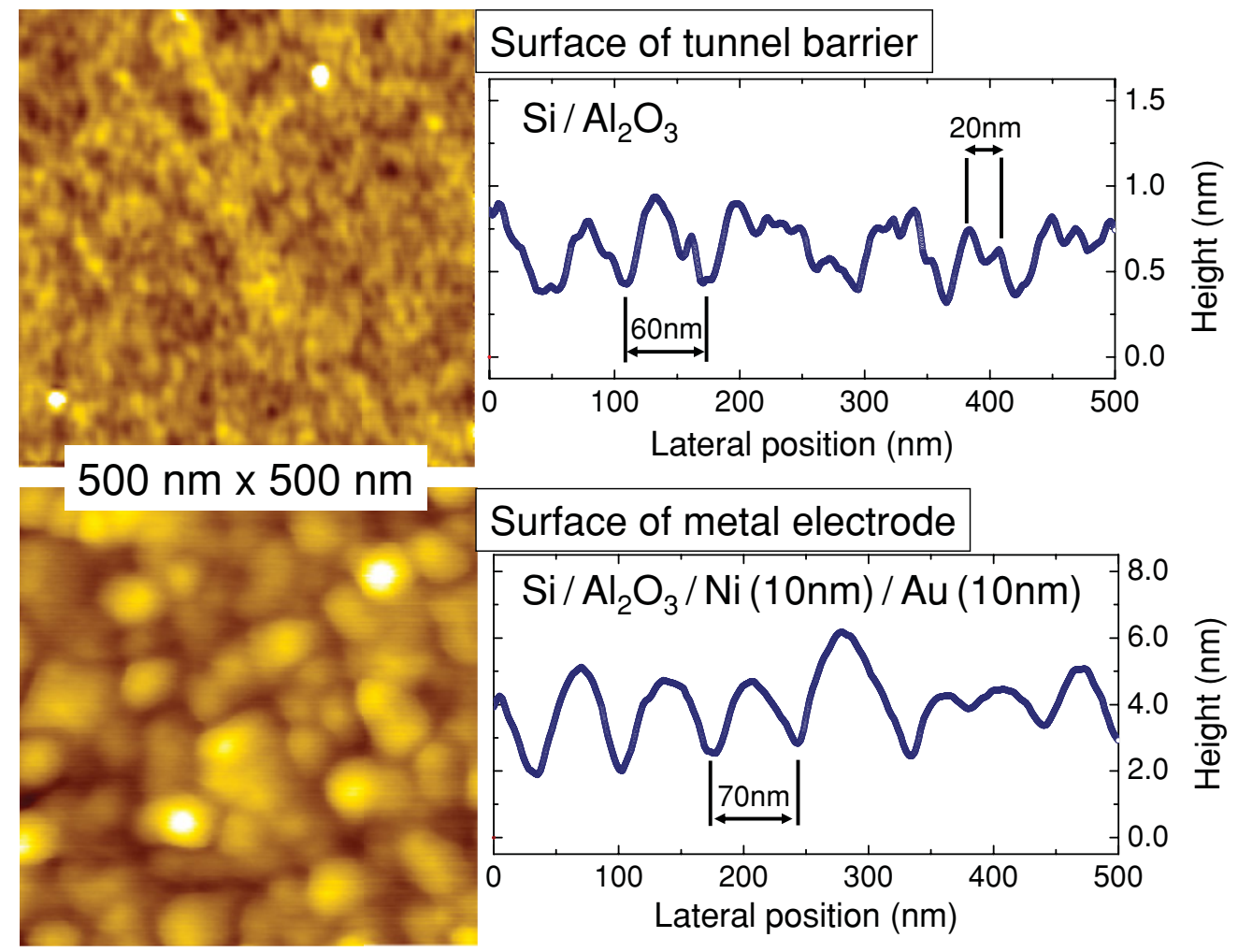

FIG. 9. (Color online) Top panels: Atomic force image $\left(500 \times 500 \mathrm{~nm}^{2}\right)$ of the surface of the $\mathrm{Al}_{2} \mathrm{O}_{3}$ tunnel barrier on $p$-type $\mathrm{Si}$, prior to metal electrode deposition, and a representative cross-sectional height profile. Bottom panels: The same, but now after deposition of the ferromagnetic metal electrode $(\mathrm{Ni}, 10 \mathrm{~nm})$ and the Au cap layer $(10 \mathrm{~nm})$.

\section{APPENDIX D: ROUGHNESS CHARACTERIZATION OF DEVICES}

Since the magnitude of the local magnetostatic field near a ferromagnetic interface depends on the amplitude and lateral period of the roughness, we performed characterization of the roughness using atomic force microscopy (AFM) under ambient conditions for some of the devices (Fig. 9). The top panel shows an AFM image of the surface of the $\mathrm{Al}_{2} \mathrm{O}_{3}$ tunnel barrier on $p$-type $\mathrm{Si}$, prior to deposition of the metal electrode. The root-mean-square (rms) roughness is about $0.2 \mathrm{~nm}$. An example of a cross-sectional height profile (right) reveals that the peak-to-peak roughness $h$ is about $0.5 \mathrm{~nm}$, while the lateral variation has two different length scales of about 20 and $60 \mathrm{~nm}$, respectively. This roughness is then copied to the bottom surface of the ferromagnetic metal that is grown on top of the tunnel barrier. The observed roughness can certainly cause local magnetostatic fields in the range of $1 \mathrm{kOe}$ to 100 Oe up to a distance of $10 \mathrm{~nm}$ away from the surface of the ferromagnet.
Because magnetostatic fields are long range and the ferromagnet is a thin film (thickness $\sim 10 \mathrm{~nm}$ ), the local magnetostatic fields that penetrate into the semiconductor are determined not only by the roughness of the bottom interface of the ferromagnet with the $\mathrm{Al}_{2} \mathrm{O}_{3}$ tunnel barrier, but also by the roughness of the top surface of the ferromagnetic layer. Unfortunately, oxidation of the ferromagnet's surface prevents a good ex situ measurement under ambient conditions, and hence no data on this are available. For the sake of completeness we did perform AFM analysis of the top of the complete metal electrode stack, consisting of $10 \mathrm{~nm}$ $\mathrm{Ni}$ and a $10 \mathrm{~nm} \mathrm{Au}$ cap layer (bottom panels of Fig. 9), although this may not be representative of the roughness of the top surface of the FM. The roughness amplitude is significantly larger (rms roughness of $1.4 \mathrm{~nm}$ and a peak-topeak amplitude of 3-4 $\mathrm{nm}$ ) compared to the surface of the tunnel barrier, while there is no small-scale $(20 \mathrm{~nm})$ lateral roughness.
${ }^{1}$ S. A. Wolf, D. D. Awschalom, R. A. Buhrman, J. M. Daughton, S. von Molnar, M. L. Roukes, A. Y. Chtchelkanova, and D. M. Treger, Science 294, 1488 (2001).

${ }^{2}$ C. Chappert, A. Fert, and F. N. Van Dau, Nature Mater. 6, 813 (2007).

${ }^{3}$ J. Fabian, A. Matos-Abiague, C. Ertler, P. Stano, and I. Žutić, Acta Phys. Slov. 57, 565 (2007).
${ }^{4}$ M. N. Baibich, J. M. Broto, A. Fert, F. Nguyen Van Dau, F. Petroff, P. Etienne, G. Creuzet, A. Friederich, and J. Chazelas, Phys. Rev. Lett. 61, 2472 (1988).

${ }^{5}$ G. Binasch, P. Grünberg, F. Saurenbach, and W. Zinn, Phys. Rev. B 39, 4828 (1989).

${ }^{6}$ J. C. Slonczewski, J. Magn. Magn. Mater. 159, L1 (1996).

${ }^{7}$ L. Berger, Phys. Rev. B 54, 9353 (1996). 
${ }^{8}$ S. Datta and B. Das, Appl. Phys. Lett. 56, 665 (1990).

${ }^{9}$ A. Fert, J.-M. George, H. Jaffrès, and R. Mattana, IEEE Trans. Electron. Devices 54, 921 (2007).

${ }^{10}$ F. J. Jedema, A. T. Filip, and B. J. van Wees, Nature (London) 410, 345 (2001).

${ }^{11}$ F. J. Jedema, H. B. Heersche, A. T. Filip, J. J. A. Baselmans, and B. J. van Wees, Nature (London) 416, 713 (2002).

${ }^{12}$ X. Lou, C. Adelmann, S. A. Crooker, E. S. Garlid, J. Zhang, K. S. M. Reddy, S. D. Flexner, C. J. Palmstrom, and P. A. Crowell, Nature Phys. 3, 197 (2007).

${ }^{13}$ B. T. Jonker, G. Kioseoglou, A. T. Hanbicki, C. H. Li, and P. E. Thompson, Nature Phys. 3, 542 (2007).

${ }^{14}$ O. M. J. van't Erve, A. T. Hanbicki, M. Holub, C. H. Li, C. Awo-Affouda, P. E. Thompson, and B. T. Jonker, Appl. Phys. Lett. 91, 212109 (2007).

${ }^{15}$ M. Ciorga, A. Einwanger, U. Wurstbauer, D. Schuh, W. Wegscheider, and D. Weiss, Phys. Rev. B 79, 165321 (2009).

${ }^{16}$ L. E. Hueso et al., Nature (London) 445, 410 (2007).

${ }^{17}$ N. Tombros, C. Jozsa, M. Popinciuc, H. T. Jonkman, and B. J. van Wees, Nature (London) 448, 571 (2007).

${ }^{18}$ M. Tran, H. Jaffrès, C. Deranlot, J.-M. George, A. Fert, A. Miard, and A. Lemaître, Phys. Rev. Lett. 102, 036601 (2009).

${ }^{19}$ Y. Ando, K. Hamaya, K. Kasahara, Y. Kishi, K. Ueda, K. Sawano, T. Sadoh, and M. Miyao, Appl. Phys. Lett. 94, 182105 (2009).

${ }^{20}$ T. Sasaki, T. Oikawa, T. Suzuki, M. Shiraishi, Y. Suzuki, and K. Tagami, Appl. Phys. Express 2, 053003 (2009).

${ }^{21}$ S. P. Dash, S. Sharma, R. S. Patel, M. P. de Jong, and R. Jansen, Nature (London) 462, 491 (2009).

${ }^{22}$ R. Jansen, B. C. Min, and S. P. Dash, Nature Mater. 9, 133 (2010).

${ }^{23}$ A. Fert and H. Jaffrès, Phys. Rev. B 64, 184420 (2001).

${ }^{24}$ V. V. Osipov and A. M. Bratkovsky, Phys. Rev. B 72, 115322 (2005).

${ }^{25}$ H. Dery and L. J. Sham, Phys. Rev. Lett. 98, 046602 (2007).

${ }^{26}$ A. N. Chantis, K. D. Belashchenko, D. L. Smith, E. Y. Tsymbal, M. van Schilfgaarde, and R. C. Albers, Phys. Rev. Lett. 99, 196603 (2007).

${ }^{27}$ Y. Song and H. Dery, Phys. Rev. B 81, 045321 (2010).
${ }^{28}$ M. Shiraishi et al., Adv. Funct. Mater. 19, 3711 (2009).

${ }^{29}$ G. Salis, A. Fuhrer, R. R. Schlittler, L. Gross, and S. F. Alvarado, Phys. Rev. B 81, 205323 (2010).

${ }^{30}$ R. I. Dzhioev, B. P. Zakharchenya, P. A. Ivanov, and L. Korenev, Pis'ma Zh. Eksp. Teor. Fiz. 60, 650 (1994) [JETP Lett. 60, 661 (1994)].

${ }^{31}$ V. L. Korenev, Semicond. Sci. Technol. 23, 114012 (2008).

${ }^{32}$ I. A. Merkulov, Al. L. Efros, and M. Rosen, Phys. Rev. B 65, 205309 (2002).

${ }^{33}$ R. I. Dzhioev, V. L. Korenev, I. A. Merkulov, B. P. Zakharchenya, D. Gammon, Al. L. Efros, and D. S. Katzer, Phys. Rev. Lett. 88, 256801 (2002).

${ }^{34}$ J. Strand, B. D. Schultz, A. F. Isakovic, C. J. Palmstrom, and P. A. Crowell, Phys. Rev. Lett. 91, 036602 (2003).

${ }^{35}$ S. Demokritov, E. Tsymbal, P. Grünberg, W. Zinn, and I. K. Schuller, Phys. Rev. B 49, 720 (1994).

${ }^{36}$ A. Brataas, G. E. W. Bauer, and P. J. Kelly, Phys. Rep. 427, 157 (2006).

${ }^{37}$ R. Jansen, B. C. Min, S. P. Dash, S. Sharma, G. Kioseoglou, A. T. Hanbicki, O. M. J. van't Erve, P. E. Thompson, and B. T. Jonker, Phys. Rev. B 82, 241305 (2010).

${ }^{38}$ B. C. Min, K. Motohashi, J. C. Lodder, and R. Jansen, Nature Mater. 5, 817 (2006).

${ }^{39}$ C. Gould, C. Ruster, T. Jungwirth, E. Girgis, G. M. Schott, R. Giraud, K. Brunner, G. Schmidt, and L. W. Molenkamp, Phys. Rev. Lett. 93, 117203 (2004).

${ }^{40}$ A. Matos-Abiague and J. Fabian, Phys. Rev. B 79, 155303 (2009).

${ }^{41}$ A. Einwanger, M. Ciorga, U. Wurstbauer, D. Schuh, W. Wegscheider, and D. Weiss, Appl. Phys. Lett. 95, 152101 (2009).

${ }^{42}$ J. C. S. Kools, W. Kula, D. Mauri, and T. Lin, J. Appl. Phys. 85, 4466 (1999).

${ }^{43}$ B. D. Schrag, A. Anguelouch, S. Ingvarsson, G. Xiao, Y. Lu, P. L. Trouilloud, A. Gupta, R. A. Wanner, W. J. Gallagher, P. M. Rice, and S. S. P. Parkin, Appl. Phys. Lett. 77, 2373 (2000).

${ }^{44}$ Optical Orientation, edited by B. P. Zakharchenya and F. Meyer (Elsevier, Amsterdam, 1984).

${ }^{45}$ A. Nogaret, J. Phys.: Condens. Matter 22, 253201 (2010).

${ }^{46}$ I. Žutić, J. Fabian, and S. Das Sarma, Rev. Mod. Phys. 76, 323 (2004). 